\title{
WMH and long-term outcomes in ischemic stroke
}

\section{A systematic review and meta-analysis}

Marios K. Georgakis, MD, Marco Duering, MD, Joanna M. Wardlaw, MD, and Martin Dichgans, MD

Neurology ${ }^{\circledR}$ 2019;92:e1298-e1308. doi:10.1212/WNL.0000000000007142

\section{Abstract}

\section{Objective}

To investigate the relationship between baseline white matter hyperintensities (WMH) in patients with ischemic stroke and long-term risk of dementia, functional impairment, recurrent stroke, and mortality.

\section{Methods}

Following the Meta-analysis of Observational Studies in Epidemiology and Preferred Reporting Items for Systematic Reviews and Meta-Analyses guidelines (PROSPERO protocol: CRD42018092857), we systematically searched Medline and Scopus for cohort studies of ischemic stroke patients examining whether MRI- or CT-assessed WMH at baseline are associated with dementia, functional impairment, recurrent stroke, and mortality at 3 months or later poststroke. We extracted data and evaluated study quality with the Newcastle-Ottawa scale. We pooled relative risks (RR) for the presence and severity of WMH using randomeffects models.

\section{Results}

We included 104 studies with 71,298 ischemic stroke patients. Moderate/severe WMH at baseline were associated with increased risk of dementia (RR 2.17, 95\% confidence interval [CI] 1.72-2.73), cognitive impairment (RR 2.29, 95\% CI 1.48-3.54), functional impairment (RR 2.21, 95\% CI 1.83-2.67), any recurrent stroke (RR 1.65, 95\% CI 1.36-2.01), recurrent ischemic stroke (RR 1.90, 95\% CI 1.26-2.88), all-cause mortality (RR 1.72, 95\% CI 1.47-2.01), and cardiovascular mortality (RR 2.02, 95\% CI 1.44-2.83). The associations followed doseresponse patterns for WMH severity and were consistent for both MRI- and CT-defined $\mathrm{WMH}$. The results remained stable in sensitivity analyses adjusting for age, stroke severity, and cardiovascular risk factors, in analyses of studies scoring high in quality, and in analyses adjusted for publication bias.

\section{Conclusions}

Presence and severity of WMH are associated with substantially increased risk of dementia, functional impairment, stroke recurrence, and mortality after ischemic stroke. WMH may aid clinical prognostication and the planning of future clinical trials.

\author{
Correspondence \\ Dr. Dichgans \\ martin.dichgans@ \\ med.uni-muenchen.de
}




\section{Glossary}

CI = confidence interval; DSM = Diagnostic and Statistical Manual of Mental Disorders; FLAIR = fluid-attenuated inversion recovery; ICH = intracerebral hemorrhage; $\mathbf{m R S}=$ modified Rankin Scale; NIHSS = NIH Stroke Scale; OR = odds ratio; $\mathbf{R C T}=$ randomized controlled trial; $\mathbf{R R}=$ relative risk; $\mathbf{W M H}=$ white matter hyperintensities.

White matter lesions identified as areas of increased signal on T2weighted and fluid-attenuated inversion recovery (FLAIR) MRI sequences or decreased signal on CT (for simplicity, termed white matter hyperintensities $[\mathrm{WMH}]$ ) are the most common imaging feature of cerebral small vessel disease, a major health problem in aging societies. ${ }^{1-3}$ The prevalence of WMH increases substantially with age $\mathrm{e}^{4,5}$ and with the presence of cardiovascular risk factors. ${ }^{6,7}$ In population-based cohort studies, $\mathrm{WMH}$ were independently associated with adverse outcomes including stroke, ${ }^{8,9}$ dementia, ${ }^{8,9}$ functional disability, ${ }^{10}$ and mortality. ${ }^{8,9}$

WMH are even more common in patients with ischemic stroke than in the general population. ${ }^{11,12}$ Brain imaging is recommended in all patients with suspected stroke. ${ }^{13}$ Hence, precise knowledge of the prognostic role of WMH may have wide clinical implications. A growing number of studies suggest that $\mathrm{WMH}$ in ischemic stroke patients are independently associated with poor outcomes including dementia, ${ }^{14-16}$ functional disability, ${ }^{17-19}$ stroke recurrence, ${ }^{20-22}$ and death. ${ }^{23-25}$ However, the reported results vary between studies possibly because of small sample size in the majority of studies, variable methodology for the assessment of WMH, variable definitions of outcomes, and different follow-up intervals. Hence, the predictive role of $\mathrm{WMH}$ after ischemic stroke remains to be defined.

Here, leveraging data from published literature, we set out to explore the long-term prognostic significance of WMH in patients with ischemic stroke in a systematic review and metaanalysis. Specifically, we aimed to assess the association between $\mathrm{WMH}$ at the time of the index stroke with long-term risk of dementia, functional impairment, recurrent stroke, and mortality.

\section{Methods}

\section{Standard protocol approvals, registrations, and patient consent}

This systematic review was based on a predefined protocol (PROSPERO registration number: CRD42018092857) following the Meta-analysis of Observational Studies in Epidemiology (MOOSE) guidelines ${ }^{26}$ and the Preferred Reporting Items for Systematic Reviews and Meta-Analyses (PRISMA). ${ }^{27}$ As all analyses have been based on publicly available summary statistics and not individual-level data, no ethical approval from an institutional review board or informed patient consent were required.

\section{Search strategy}

We systematically searched Medline and Scopus from inception through June 1, 2018 (detailed search strategy in
e-Methods, doi.org/10.5061/dryad.8b62gn1), screened ProQuest and OpenGrey as sources of gray literature, and handsearched the reference lists of eligible articles and relevant reviews without language or publication year restrictions. We evaluated studies for potential population overlap, based on geographical setting and recruitment period. In case of overlap, we included studies presenting the most fully adjusted model or the ones with the largest sample size. Two authors (M. Georgakis and M. Duering) performed the literature search independently, and differences were resolved through consensus.

\section{Inclusion criteria}

We considered eligible all prospective or retrospective cohort studies that included patients with ischemic stroke and examining the association of WMH at baseline with the outcomes of interest over a follow-up period of $\geq 3$ months. Secondary analyses of randomized controlled trials (RCTs) examining the same outcomes were also included. Casecontrol studies, cross-sectional studies, case reports, case series of $<50$ patients, and animal studies were excluded. Our target population was adult ( $\geq 18$ years) patients with ischemic stroke. Studies examining exclusively patients with hemorrhagic stroke and patients with TIA were excluded. However, we included studies examining mixed populations of patients with ischemic stroke and TIA or hemorrhagic stroke, as ischemic stroke patients usually comprise the majority of participants in these cohorts.

In order to qualify for inclusion into the meta-analysis, WMH had to be assessed within the first 3 months after the index stroke event. We included studies assessing WMH by either MRI or CT, as previous studies have shown substantial agreement between the 2 methods. ${ }^{28,29}$ We further included both studies assessing WMH severity through semiquantitative visual rating methods and studies with quantitative measurements of $\mathrm{WMH}$ volume.

Our primary outcomes included poststroke dementia, functional impairment, any recurrent stroke, and all-cause mortality, assessed over a period of $\geq 3$ months after stroke. Dementia had to be defined by standardized criteria (e.g., DSM) or validated clinical rating scales (e.g., Clinical Dementia Rating scale). Cognitive impairment (mild cognitive impairment or dementia), defined by formal neuropsychological testing or global cognitive tests with validated cutoffs, was examined as a secondary outcome. Studies examining performance in specific cognitive domains in continuous scales were excluded. For functional outcome, we included studies using tools that have specifically been 
validated to determine disability in the poststroke setting, such as modified Rankin Scale (mRS), the Oxford Handicap Scale, ${ }^{30}$ the Barthel Index, ${ }^{31}$ and tools assessing functionality in activities of daily living. ${ }^{32}$ Functional impairment defined by a validated cutoff with any of these tools was our primary outcome, but we also separately examined poor functional outcome, defined by an mRS score of $>2$ or $>1$. Studies not dichotomizing functional outcome or not providing data allowing its dichotomization were excluded. For recurrent stroke, we considered studies assessing any recurrent stroke (ischemic or hemorrhagic) through imaging or clinical followup or through linkage with disease registries. Recurrent ischemic stroke was a secondary outcome. We included studies assessing all-cause mortality through assessment of death certificates, linkage with national death registry data, or via interview with informants during follow-up. Cardiovascular mortality including death due to ischemic heart disease or stroke was a secondary outcome.

\section{Quality assessment}

We evaluated study quality using the cohort subscale of the

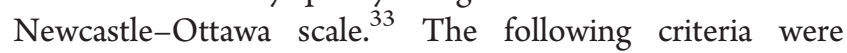
assessed: representativeness of exposed cohort, selection of nonexposed cohort, exposure ascertainment, outcome presence at study onset, comparability of exposed and unexposed cohorts, outcome assessment, follow-up length, and follow-up adequacy. Quality score ranges from 0 to 9 points. Quality assessment is described in detail in the e-Methods (doi.org/ 10.5061 /dryad.8b62gn1).

\section{Data extraction}

We used a predefined spreadsheet to extract the following information from each article: study characteristics (geographical setting, recruitment period, study design); population details (sample size, inclusion/exclusion criteria, stroke subtypes, age, sex, stroke severity, cardiovascular risk factors); WMH ascertainment (imaging modality, method and scale of quantification); outcome assessment (definition, follow-up timepoint, number of events); and statistical analysis (type of analysis, relative risk [RR], 95\% confidence intervals [CIs], adjustments). If multiple analysis models were presented in the individual articles, we extracted the RRs from the most fully adjusted model. Studies examining cognitive outcomes, recurrent stroke, and mortality presented hazard ratios or rate ratios as effect estimates of $R R$, which were combined in the same meta-analyses, whereas studies examining functional outcome presented odds ratios (OR). When the effect estimate was not directly provided, we calculated RRs or ORs, respectively, through $2 \times 2$ tables. To address the time dependency of cognitive outcomes, recurrent stroke, and mortality, we also performed sensitivity analyses restricted to hazard ratios.

\section{Statistical analysis}

Our predefined primary analysis approach was a comparison of moderate/severe vs mild/none $\mathrm{WMH}$, given the high prevalence of mild WMH alterations in imaging studies of elderly individuals. ${ }^{4}$ To harmonize studies using different $\mathrm{WMH}$ rating scales for defining moderate/severe $\mathrm{WMH}$, we used the definition suggested and validated for each individual scale. For studies assessing WMH volume and presenting results in quantiles, we set a cutoff above the median quantile to define moderate/severe WMH. When studies presented RRs for $>1 \mathrm{WMH}$ severity categories, we obtained the effect estimate for moderate/severe vs mild/none categories using the method suggested by Hamling et al. ${ }^{34}$ We also performed alternative analyses comparing presence vs absence of $\mathrm{WMH}$ and continuous analyses for increasing WMH severity. Studies presenting continuous analyses on different severity scale ranges were harmonized to a 0-3 scale. Studies presenting continuous analyses for WMH volume were analyzed separately.

To account for the differences across studies regarding WMH assessment, we pooled the effect sizes of different studies using random-effects meta-analyses. We set our Bonferronicorrected significance threshold to $p=0.05 / 4=0.0125$ to correct for the 4 different primary outcomes. We evaluated between-study heterogeneity with the $I^{2}$ and the Cochran $Q$ statistic; $I^{2}$ exceeding $50 \%$ or $75 \%$ was considered as moderate and high heterogeneity, respectively. ${ }^{35}$ We evaluated publication bias using the Egger test (significance threshold set at $p$ $<0.10),{ }^{36}$ and adjusted the pooled effect estimates for publication bias in a "trim and fill" analysis. ${ }^{37}$

Sensitivity analyses were performed for the different imaging modalities (MRI/CT) and WMH quantification methods (semi-quantitative/volumetry). Additional subgroup analyses were conducted by study design (prospective/retrospective and after excluding RCTs), study population (solely ischemic stroke/ischemic stroke plus TIA or intracerebral hemorrhage $[\mathrm{ICH}]$ ), degree of confounder adjustment (age, stroke severity, cardiovascular risk factors), stroke severity (NIH Stroke Scale [NIHSS] score), age ( $<70 / \geq 70$ years), WMH location (periventricular/deep), MRI sequence (FLAIR/T2), follow-up duration $(<1 / \geq 1$ year), sum quality score, and fulfillment of every quality item. In case of heterogeneity, we examined whether it was explained by any of these factors. We further conducted meta-regression analysis to evaluate whether stroke subtypes, age, sex, education, NIHSS score, atrial fibrillation, hypertension, hypercholesterolemia, diabetes mellitus, smoking, stroke history, and coronary artery disease modified the examined associations.

For studies presenting WMH analyses in $\geq 3$ levels of $\mathrm{WMH}$ severity and adjusting their results at least for age, we conducted dose-response meta-analyses for our primary outcomes. To assign "doses" to every WMH category, we harmonized every scale to a $0-3$ severity scale (none, mild, moderate, severe). ${ }^{38}$ We applied restricted cubic spline models, using generalized least square regression with predefined knots at 10th, 50th, and 90th percentiles, for individual studies and thereafter pooled the study-specific estimates using the restricted maximum likelihood method in 
a random-effects meta-analysis. ${ }^{39}$ All statistical analyses were conducted in Stata 13.1 (StataCorp, College Station, TX).

\section{Data availability}

The analysis for this study is based on published results from individual studies. Therefore, individual-level data cannot be made publicly available. All extracted data from the individual studies and the code used for performing the meta-analyses can be made available upon reasonable request to the corresponding author.

\section{Results}

\section{Review of literature}

Figure 1 illustrates the study selection process. The literature search yielded 6,562 articles that were screened for eligibility. Following evaluation of titles and abstracts, we examined the full text of 248 articles for eligibility. We excluded 128 articles that did not meet our eligibility criteria (table e-1, doi.org/10.5061/dryad.8b62gn1) and 16 articles because of overlap with other eligible articles (table e-2, doi.org/10.5061/dryad.8b62gn1), leaving 104 articles for inclusion into our systematic review. Thirty-one articles ( $\mathrm{n}=16,167$ individuals) assessed cognitive outcomes, 49 articles $(n=25,559)$ assessed functional outcomes, 26 articles $(n=30,256)$ assessed recurrent stroke, and 28 articles $(\mathrm{n}=15,533)$ assessed mortality.

\section{Study characteristics}

The characteristics of studies eventually included in the final analysis are presented in table e-3 (doi.org/10.5061/dryad. 8 b62gn1). Overall, the 104 studies included a total of 71,298 individuals (median sample size $\mathrm{n}=266$ individuals; minimum $\mathrm{n}=56$; maximum $\mathrm{n}=9,522$ ). Forty-four studies were retrospective cohort studies $(n=20,765)$, whereas 60 had a prospective design $(n=50,533)$. Of the latter, 3 studies presented results from secondary analyses of RCTs $(n=6,637)$. The majority of studies (101 out of 104 studies; $n=67,769$ ) were hospital-based and examined older stroke patients (mean age $\geq 65$ years in 82 studies; $n=62,304)$. Thirty-eight studies $(n=$ 33,067 ) included TIA or ICH patients in addition to ischemic stroke patients, whereas 33 studies $(n=6,278)$ focused on specific ischemic stroke subgroups, mainly lacunar stroke or mild stroke. WMH were assessed solely through MRI in 66 studies $(\mathrm{n}=47,963)$, solely through CT in 31 studies $(\mathrm{n}=$ $17,144)$, and through either method in 7 studies $(n=6,191)$. Regarding WMH quantification, 10 studies $(\mathrm{n}=7,027)$ used volumetry, whereas 77 studies $(n=56,017)$ rated $\mathrm{WMH}$ severity with visual semi-quantitative rating scales, the most common of which were the Fazekas scale (40 studies; $\mathrm{n}=$ 39,776 ), the Van Swieten scale ( 15 studies; $n=6,977$ ), and the age-related white matter changes (ARWMC) scale (15 studies; $\mathrm{n}=8,248)$. The remaining 17 studies $(\mathrm{n}=8,254)$ assessed WMH presence only through subjective visual assessment. Follow-up ranged from 3 months to 15 years with mean intervals of 38.3 months for cognitive outcomes, 5.8 months for functional outcomes, 34.3 months for recurrent stroke, and 33.1 months for mortality.

\section{Study quality}

The overall study quality was moderate, with only 7 studies (7\%) fulfilling all quality items assessed by the NewcastleOttawa scale (table e-4, doi.org/10.5061/dryad.8b62gn1). Median total quality scores for cognitive outcomes, functional outcomes, recurrent stroke, and mortality were 5/9, 7/9, 7/9, and $5 / 8$, respectively. Regarding the items on selection bias, 33 studies (32\%) lost quality points in representativeness of the cohort, 17 studies (16\%) because of nonvalidated subjective assessment of $\mathrm{WMH}$, and 57 studies (55\%) for not ensuring absence of the outcome at study onset. Furthermore, 38 studies (37\%) did not adjust their results for age and 41 studies (39\%) did not adjust for cardiovascular risk factors or stroke severity. Regarding outcome assessment, 16 studies (52\%) did not define dementia or cognitive impairment by standardized clinical criteria and formal neuropsychological assessment, 10 studies (38\%) did not confirm recurrent stroke with imaging, and 16 studies (57\%) examining mortality did not cross-link deaths with death certificates. Follow-up interval was considered short ( $<12$ months) for 18 studies (58\%) on cognitive outcome, 6 studies $(23 \%)$ on recurrent stroke, and 10 studies (36\%) on mortality. Finally, 61 studies (59\%) did not meet the quality criteria for follow-up adequacy, as the attrition rates were $>10 \%$.

\section{WMH and outcomes after stroke}

We found moderate/severe WMH at baseline (figure 2A) to be associated with increased risk of dementia (RR 2.17, 95\% CI 1.72-2.73; 12 studies; 12,341 individuals; 2,159 events), any functional impairment (RR 2.21, 95\% CI 1.83-2.67; 25 studies; 16,339 individuals; 6,663 events), any recurrent stroke (RR 1.65, 95\% CI 1.36-2.01; 14 studies; 24,166 individuals; 3,168 events), and all-cause mortality (RR 1.72, 95\% CI 1.47-2.01; 15 studies; 11,553 individuals; 1,801 events) after ischemic stroke. Our analyses further showed that WMH were associated with all of the examined secondary outcomes including cognitive impairment (RR 2.29, 95\% CI 1.48-3.54; 8 studies; 9,505 individuals; 1,299 events), $\mathrm{mRS}>1$ (RR 1.96, 95\% CI 1.67-2.29; 14 studies; 14,155 individuals; 7,350 events), mRS >2 (RR 2.27, 95\% CI 1.84-2.81; 19 studies; 13,979 individuals; 5,899 events), recurrent ischemic stroke (RR 1.90, 95\% CI 1.26-2.88; 6 studies; 5,505 individuals; 423 events), and cardiovascular mortality (RR 2.02, 95\% CI 1.44-2.83; 5 studies; 2,499 individuals; 621 events).

The analyses examining WMH presence vs absence (figure 2B) and $\mathrm{WMH}$ severity in continuous analyses (figure $2 \mathrm{C}$ ) likewise showed consistent results for all of the examined primary and secondary outcomes, except for cardiovascular mortality, where no studies were available for pooling. Forest plots of the above results are presented in figures e-1-e-4 (doi.org/10.5061/ dryad.8b62gn1). A meta-analysis of studies examining WMH volume in linear association with any functional impairment had similar results (OR per $10 \mathrm{~mL}$ increase: 1.29, 95\% 
Figure 1 Flowchart on the selection of eligible articles

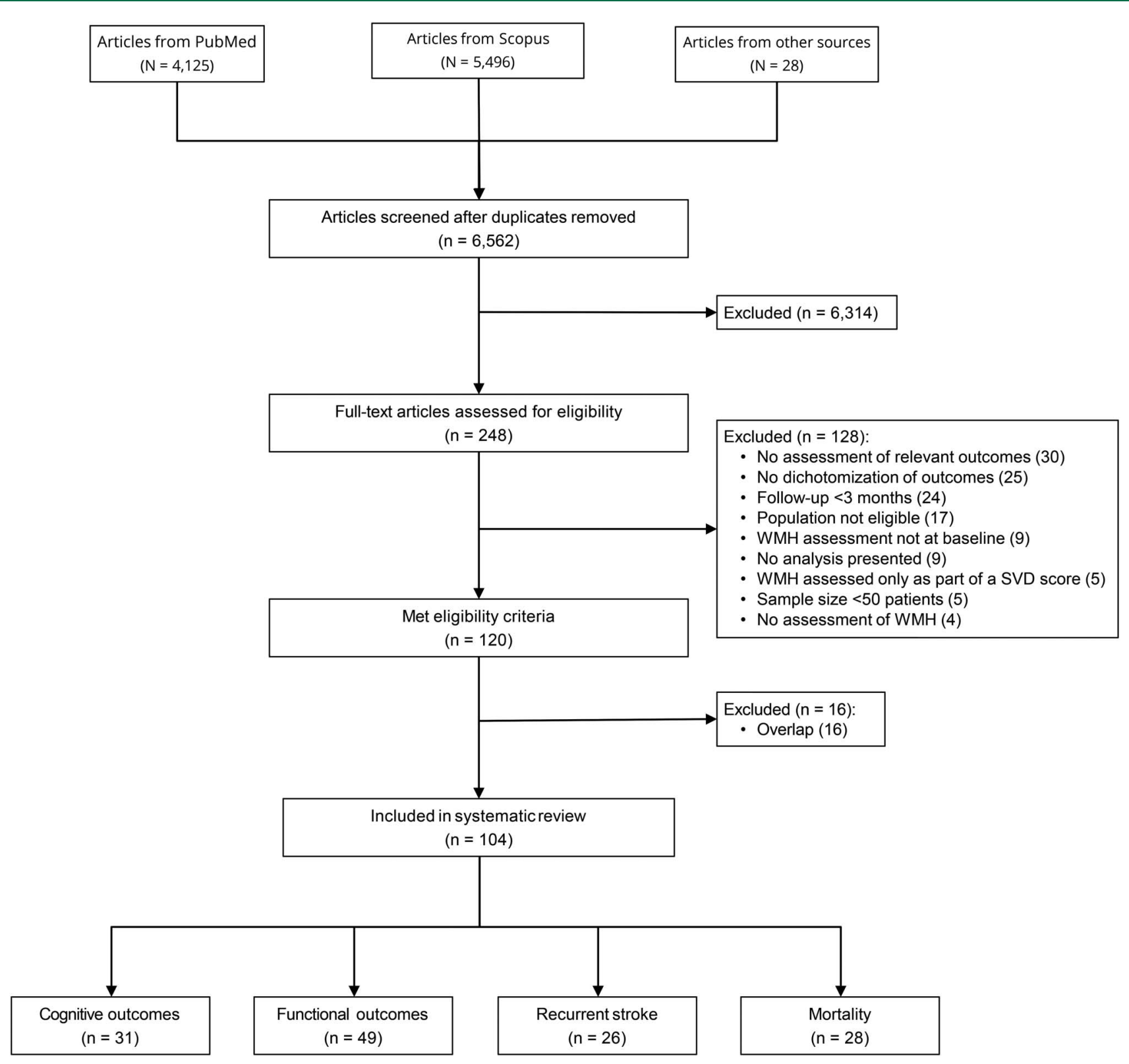

SVD = small vessel disease; $\mathrm{WMH}=$ white matter hyperintensities.

CI $1.10-1.51$ and OR per 1 log-WMH volume: 1.20 , 95\% CI 1.01-1.44) (figure e-5, doi.org/10.5061/dryad.8b62gn1).

\section{Heterogeneity and sensitivity analyses}

We found moderate to high heterogeneity in the moderate/ severe vs mild/none and in the presence vs absence analyses, but no heterogeneity was noted in the continuous WMH severity analyses (figure 2). To examine the sources of heterogeneity, we performed sensitivity analyses. Our results remained stable in sensitivity analyses restricted to studies of prospective or retrospective design, studies including solely patients with ischemic stroke, studies adjusting for age, stroke severity, or cardiovascular risk factors, studies of mean follow-up duration $<1$ or $\geq 1$ year, studies of higher quality, studies including patients $<70$ or $\geq 70$ years and of different stroke severity, studies evaluating WMH by either MRI or $\mathrm{CT}$, and in T2 or FLAIR sequences, studies quantifying WMH by volumetry or semi-quantitative scales, and studies examining periventricular or deep WMH (figure 3). Our results further remained stable in sensitivity analyses excluding studies based on secondary analyses of RCTs (figure e-6, doi.org/10.5061/dryad.8b62gn1) and when restricting the analyses to studies presenting hazard ratios as risk estimates (figure e-7, doi.org/10.5061/dryad.8b62gn1).

Interestingly, sensitivity analyses restricted to studies assessing WMH through FLAIR MRI were not accompanied by heterogeneity for any of the examined outcomes. Also, 
Figure 2 Associations between white matter hyperintensities (WMH) at baseline and outcomes after stroke

A. Moderate/severe vs mild/non WMH

\begin{tabular}{|c|c|c|c|c|c|c|c|c|}
\hline \multirow[b]{2}{*}{ Outcome } & \multirow{2}{*}{$\begin{array}{l}\text { Studies } \\
\text { (k) }\end{array}$} & \multirow{2}{*}{$\begin{array}{l}\text { Events/sample } \\
\text { (N) }\end{array}$} & \multirow{2}{*}{$\begin{array}{l}\text { Follow-up } \\
\text { (mean, months) }\end{array}$} & & \multicolumn{2}{|c|}{ Effectsize } & \multicolumn{2}{|c|}{ Heterogeneity } \\
\hline & & & & & RR (95\% Cl) & $p$ & $I^{2}$ & $p$ \\
\hline \multicolumn{9}{|l|}{ Cognitive } \\
\hline Dementia & 12 & $2159 / 12341$ & 51.6 & $\rightarrow$ & $2.17(1.72,2.73)$ & $4.4 \times 10^{-11}$ & 33 & 0.12 \\
\hline Impairment & 8 & 1299/9505 & 55.8 & $\longrightarrow$ & $2.29(1.48,3.54)$ & $2.0 \times 10^{-4}$ & 65 & 0.006 \\
\hline \multicolumn{9}{|l|}{ Functional outcomes } \\
\hline Any functional impairment & 25 & $6663 / 16339$ & 4.8 & $\rightarrow$ & $2.21(1.83,2.67)$ & $1.9 \times 10^{-16}$ & 69 & $<0.001$ \\
\hline$m R S>1$ & 14 & $7350 / 14155$ & 4.5 & $\rightarrow$ & $1.96(1.67,2.29)$ & $2.2 \times 10^{-16}$ & 56 & 0.005 \\
\hline$m R S>2$ & 19 & $5899 / 13979$ & 3.9 & $\rightarrow$ & $2.27(1.84,2.81)$ & $3.7 \times 10^{-14}$ & 69 & $<0.001$ \\
\hline \multicolumn{9}{|l|}{ Recurrent stroke } \\
\hline Any recurrent & 14 & $3168 / 24166$ & 37.1 & $\rightarrow$ & $1.65(1.36,2.01)$ & $5.4 \times 10^{-7}$ & 52 & 0.04 \\
\hline Recurrent ischemic & 6 & $423 / 5505$ & 41.1 & $\longrightarrow$ & $1.90(1.26,2.88)$ & $2.0 \times 10^{-3}$ & 53 & 0.06 \\
\hline \multicolumn{9}{|l|}{ Mortality } \\
\hline All-cause & 15 & $1801 / 11553$ & 27.1 & - & $1.72(1.47,2.01)$ & $1.1 \times 10^{-9}$ & 46 & 0.03 \\
\hline Cardiovascular & 5 & $621 / 2499$ & 66.2 & $\rightarrow$ & $2.02(1.44,2.83)$ & $4.3 \times 10^{-5}$ & 69 & 0.01 \\
\hline
\end{tabular}

B. Presence vs absence of WMH

\begin{tabular}{|c|c|c|c|c|c|c|c|c|}
\hline \multirow[b]{2}{*}{ Outcome } & \multirow{2}{*}{$\begin{array}{l}\text { Studies } \\
\text { (k) }\end{array}$} & \multirow{2}{*}{$\begin{array}{l}\text { Events/sample } \\
\text { (N) }\end{array}$} & \multirow{2}{*}{$\begin{array}{l}\text { Follow-up } \\
\text { (mean, months) }\end{array}$} & & \multicolumn{2}{|l|}{ Effectsize } & \multicolumn{2}{|c|}{ Heterogeneity } \\
\hline & & & & & RR $(95 \% \mathrm{Cl})$ & $p$ & $1^{2}$ & $p$ \\
\hline \multicolumn{9}{|l|}{ Cognitive } \\
\hline Dementia & 12 & $484 / 2938$ & 13.1 & $\rightarrow$ & $2.36(1.74,3.20)$ & $2.9 \times 10^{-8}$ & 4.5 & 0.4 \\
\hline Impairment & 7 & $419 / 721$ & 6.5 & $\rightarrow$ & $2.22(1.45,3.42)$ & $2.6 \times 10^{-4}$ & 0 & 0.74 \\
\hline \multicolumn{9}{|l|}{ Functional outcomes } \\
\hline Any functional impairment & 21 & $6369 / 13433$ & 6.6 & $\rightarrow$ & $1.96(1.62,2.37)$ & $3.9 \times 10^{-12}$ & 56 & 0.001 \\
\hline $\mathrm{mRS}>1$ & 9 & $4738 / 8291$ & 4.9 & $\rightarrow$ & $1.73(1.44,2.09)$ & $5.2 \times 10^{9} 9$ & 37 & 0.12 \\
\hline$m R S>2$ & 12 & $5203 / 10388$ & 5.3 & $\rightarrow$ & $1.96(1.55,2.50)$ & $3.4 \times 10.8$ & 56 & 0.009 \\
\hline \multicolumn{9}{|l|}{ Recurrentstroke } \\
\hline Any recurrent & 15 & $1262 / 11753$ & 33.4 & $\rightarrow$ & $1.71(1.36,2.14)$ & $3.4 \times 10.6$ & 32 & 0.15 \\
\hline Recurrent ischemic & 5 & $363 / 4450$ & 44.1 & - & $1.93(1.01,3.69)$ & 0.047 & 66 & 0.02 \\
\hline \multicolumn{9}{|l|}{ Mortality } \\
\hline All-cause & 15 & $1401 / 11492$ & 18.2 & - & $1.82(1.51,2.19)$ & $3.0 \times 10.10$ & 56 & 0.004 \\
\hline Cardiovascular & 0 & $\%$ & - & & - & - & - & - \\
\hline & & & $\begin{array}{r}1 \\
0.25\end{array}$ & & & & & \\
\hline
\end{tabular}

C. 1 grade increment (scale 0-3) for WHM (continuous analysis)

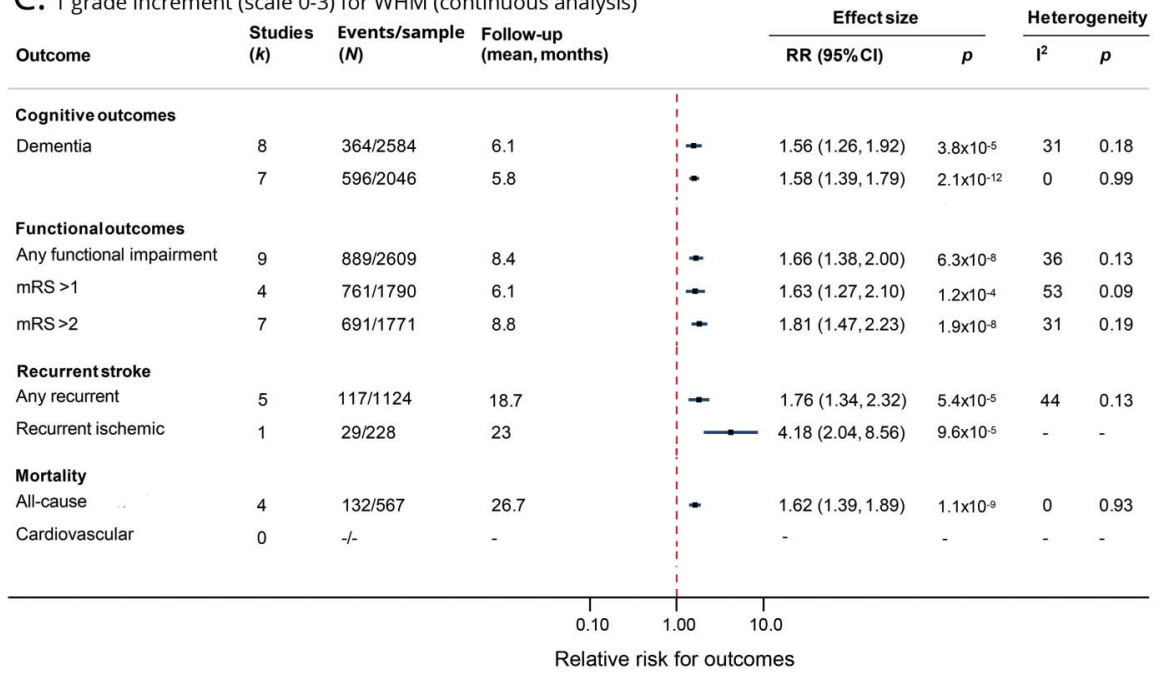

The forest plots depict the summary effect estimates for the associations of WMH with cognitive outcomes, functional outcomes, recurrent stroke, and mortality based on 3 different approaches of analyzing WMH severity ([A] moderate/severe vs mild/none $\mathrm{WMH}_{\text {; }}[\mathrm{B}]$ present vs none $\mathrm{WMH} ;[\mathrm{C}] 1$ grade increment [scale 0-3] for WMH [continuous analysis]). $\mathrm{Cl}=$ confidence interval; $\mathrm{mRS}=$ modified Rankin Scale; $R R=$ relative risk. heterogeneity for dementia, any recurrent stroke, and allcause mortality was resolved in analyses restricted to prospective studies, studies restricted to ischemic stroke patients, and studies adjusting for the maximum number of confounders. Finally, no heterogeneity was noted for mortality when restricting analyses to studies with a follow-up $\geq 1$ year (figure 3). Sensitivity analyses restricted to studies fulfilling every Newcastle-Ottawa scale quality criterion also 
Figure 3 Subgroup analyses of the association of white matter hyperintensities (WMH) at baseline with stroke outcomes across different study characteristics

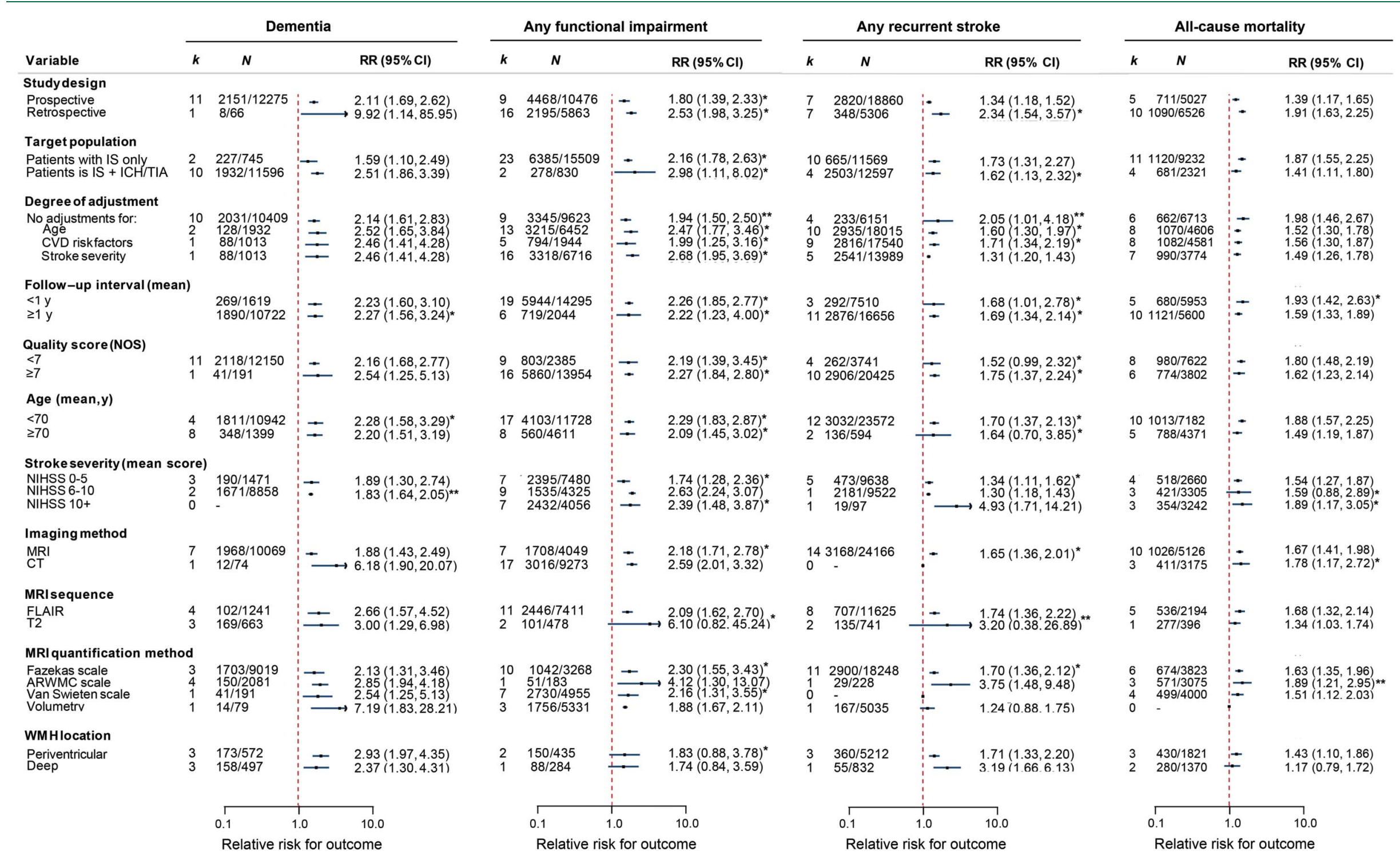

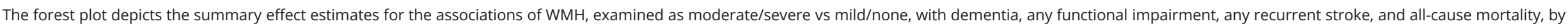

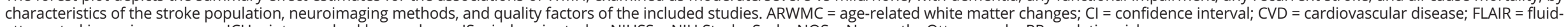
attenuated inversion recovery; ICH = intracerebral hemorrhage; IS = ischemic stroke; NIHSS = NIH Stroke Scale; NOS = Newcastle-Ottawa scale; RR = relative risk. 
Figure 4 Dose-response meta-analysis of the age-adjusted association of white matter hyperintensities severity at baseline with stroke outcomes

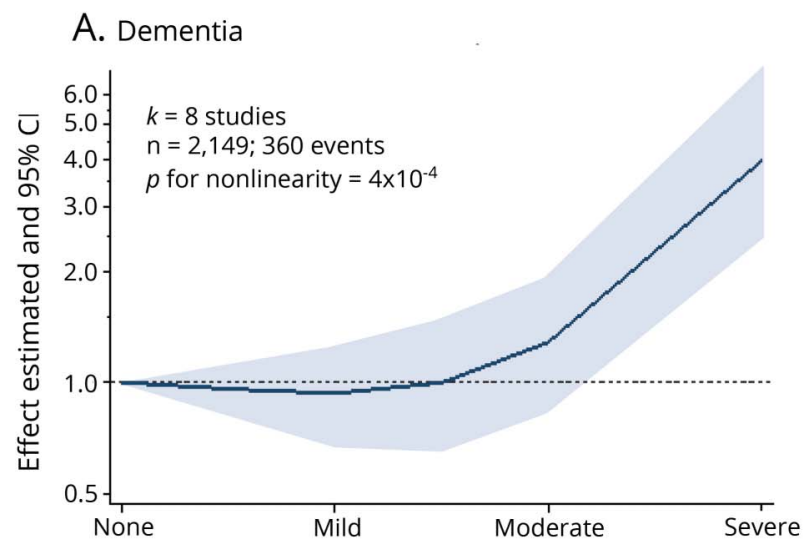

B. Any functional impairment

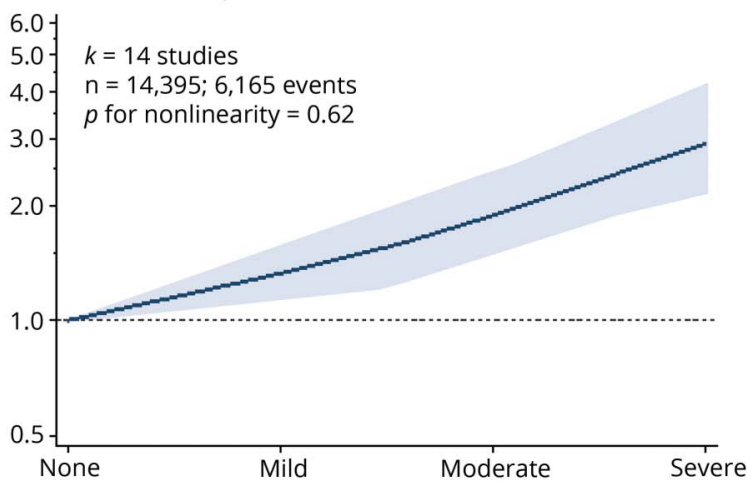

D. All-cause mortality

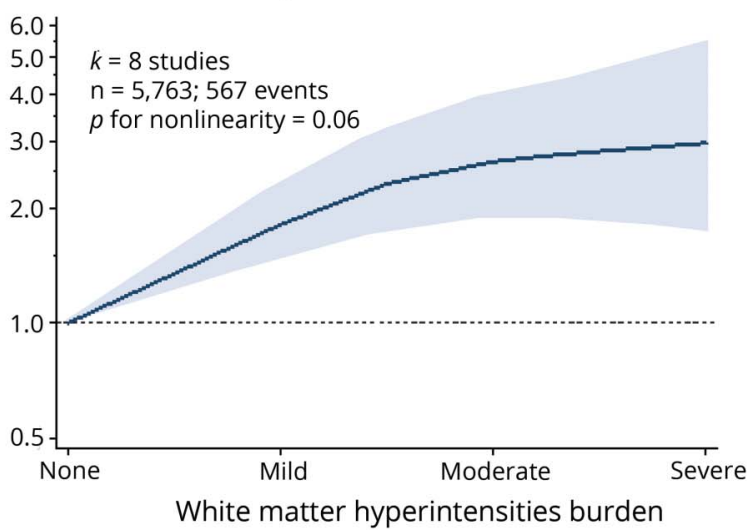

The graphs depict the restricted cubic spline derived effect estimates and their 95\% confidence intervals (Cls) for (A) dementia, (B) any functional impairment, (C) any recurrent stroke, and (D) all-cause mortality.

showed consistent associations of WMH with all of the outcomes (table e-5, doi.org/10.5061/dryad.8b62gn1). Importantly, we found no heterogeneity in analyses for dementia and recurrent stroke restricted to representative stroke populations, and to studies ensuring the absence of prestroke cognitive impairment or history of stroke at study onset, respectively (table e-5, doi.org/10.5061/ dryad.8b62gn1).

Meta-regression analyses showed that none of the examined study population characteristics or quality score modified the associations of $\mathrm{WMH}$ with stroke outcomes (table e-6, doi. org/10.5061/dryad.8b62gn1). However, there was some indication that the effect size of the association between WMH and dementia increased with an increasing proportion of small vessel stroke and decreased with an increasing proportion of cardioembolic stroke within the study population.

\section{Dose-response associations between WMH and outcomes after stroke}

We next examined dose-response associations of WMH severity with stroke outcomes (figure 4). In age-adjusted dose-response meta-analyses, we found linear associations between WMH severity and the relative risk for functional impairment $(p$ for nonlinearity $=0.62 ; 14$ studies; 14,395 individuals; 6,165 events), recurrent stroke ( $p$ for nonlinearity $=0.48 ; 8$ studies; 10,448 individuals; 668 events), and allcause mortality ( $p$ for nonlinearity $=0.06 ; 8$ studies; 5,763 individuals; 567 events), but a nonlinear association for dementia ( $p$ for nonlinearity $=4 \times 10^{-4} ; 8$ studies; 2,149 individuals; 360 events). More specifically, WMH increased the risk for dementia only above a moderate WMH severity. Restricting analyses to studies further adjusting for stroke severity and cardiovascular risk factors yielded similar results (figure e-8, doi.org/10.5061/dryad.8b62gn1).

\section{Assessment of publication bias}

The Egger test supported presence of small-study effects indicating publication bias in several of the primary analyses (table e-7 and figure e-9, doi.org/10.5061/dryad.8b62gn1). Hence, we adjusted our results for publication bias through a "trim and fill" analysis. Importantly, the effect estimates were attenuated, but the associations remained in these adjusted analyses (table e-7, doi.org/10.5061/dryad.8b62gn1). 


\section{Discussion}

Pooling data from 104 studies including $>70,000$ ischemic stroke patients, we found that WMH at the time of stroke are associated with multiple unfavorable long-term outcomes. Specifically, we found both the presence and an increasing severity of WMH to be associated with a higher risk of cognitive impairment and dementia, functional impairment, recurrent stroke, all-cause mortality, and cardiovascular mortality in follow-up intervals extending up to 15 years after stroke. The associations followed a dose-response pattern, with severe WMH being associated with the highest risk for poor long-term outcomes after stroke. The results were consistent regardless of imaging modality, were stable in sensitivity analyses of studies scoring high in quality and of studies adjusting for age, stroke severity, and cardiovascular risk factors, and remained after adjustment for publication bias.

Our findings extend previous literature on the prognostic role of WMH in the general population. ${ }^{8,9}$ Stroke marks a highrisk population amenable to preventive therapies and regular monitoring. Other than healthy elderly, almost all stroke patients receive brain imaging as part of their diagnostic workup. ${ }^{13}$ WMH can be reliably assessed on routine $\mathrm{CT}$ and MRI scans. ${ }^{28,29,40}$ As such, our finding of a predictive role of WMH for multiple long-term outcomes might have wide clinical implications. Specifically, the assessment of WMH might aid clinical prognostication, contribute to the interpretation of clinical trials, and possibly also influence treatment decisions, although this would need to be examined in controlled trials. To date, there are no treatment options with proven efficacy for slowing or halting the progression of WMH lesions. In any case, as WMH influence stroke outcomes, they should (1) be considered as a confounder for inclusion in minimization algorithms to balance baseline characteristics during randomization into trials; (2) be examined for potential interactions with treatment effect; and (3) be explored as a prognostic variable in future observational studies of patients with ischemic stroke.

The mechanisms underlying the associations of WMH with stroke outcomes are poorly understood. Potential explanations come from the following observations: first, WMH severity has been associated with infarct growth and larger infarct volumes in ischemic stroke patients, ${ }^{41-43}$ suggesting that the pathologic changes underlying WMH enhance susceptibility to acute ischemia. Indeed, WMH have been associated with both microvascular rarefaction ${ }^{44}$ and reduced blood flow ${ }^{45}$ not only in WMH, but also in normal-appearing brain tissue. Second, similar to other manifestations of small vessel disease, ${ }^{46} \mathrm{WMH}$ are a risk factor for symptomatic intracerebral hemorrhage after ischemic stroke in patients receiving thrombolysis. ${ }^{19,47}$ Third, we found that WMH increase the risk for stroke recurrence long-term, which might in part explain the association between $\mathrm{WMH}$ and cognitive outcome, functional outcome, and mortality. Of note, however, the stroke recurrence rate observed here was lower than the incidence rates for these events. Hence, the effects of $\mathrm{WMH}$ on risk of dementia, functional impairment, and mortality are not sufficiently explained by the effects on stroke recurrence. Fourth, WMH might influence poststroke outcomes by disrupting neuronal networks relevant for cognitive reserve $^{48}$ and rehabilitation. ${ }^{49}$ Finally, WMH might reflect the systemic burden of vascular risk factors known to influence stroke outcome, ${ }^{6,7}$ although the results remained stable in studies adjusting for these factors.

The identified interaction between ischemic stroke subtype and the effect of WMH on dementia risk perhaps reflects fundamental differences in mechanisms of dementia after different ischemic stroke subtypes. Specifically, we found the association between WMH burden and poststroke dementia to be stronger in studies with higher representation of small vessel stroke. This interaction could relate to aspects of infarct location involving strategic lesions in subcortical gray or white matter. ${ }^{50}$ Nevertheless, these findings are difficult to interpret in a meta-analysis setting and future original observational studies are needed to clarify the role of ischemic stroke subtypes in the examined associations.

This study has several strengths. Our systematic review was based on a predefined protocol and followed standard guidelines with rigorous screening of $>6,000$ articles including sources of gray literature and without language or publication year restrictions. Our pooled analysis was based on a large number of studies examining a wide range of stroke outcomes in more than 70,000 patients, thus providing robust estimates for the associations of WMH with dementia, functional impairment, mortality, and recurrent stroke. Finally, extensive sensitivity and meta-regression analyses enabled us to control for confounding and other forms of bias.

Our study also has limitations. First, the main analyses revealed substantial heterogeneity. Potential sources of this heterogeneity include between-study differences in target population, study design, assessment and quantification of WMH, definition and ascertainment of outcomes, follow-up duration, and statistical approaches. Second, the majority of studies were of rather lower quality. Specifically, several of the included studies were not representative of the general stroke population, showed high attrition rates, did not assess whether outcomes were present before stroke, and did not adjust for major confounders such as age, NIHSS, and cardiovascular risk factors. Third, our analyses suggest marked publication bias for all outcomes investigated. However, the associations between $\mathrm{WMH}$ and long-term outcomes remained when adjusting for publication bias. Finally, we could not examine the influence of the index infarct on the technical assessment of WMH and whether this affected the results.

This meta-analysis shows that in patients with ischemic stroke, both the presence and extent of WMH are associated 
with substantially increased risk of multiple long-term outcomes including dementia, functional impairment, recurrent stroke, and mortality. Our findings may have implications for clinical prognostication and the planning and interpretation of clinical trials.

\section{Study funding}

Vascular Dementia Research Foundation; Deutsche Forschungsgemeinschaft (German Research Foundation); Munich Cluster for Systems Neurology (EXC 1010 SyNergy); European Union's Horizon 2020 research and innovation programmes under grant agreements 666,881 (SVDs@target) and 667,375 (CoSTREAM); German Center for Neurodegenerative Diseases (DZNE). The funding organizations had no role in the design or conduct of the study; collection, management, analysis, or interpretation of the data; preparation, review, or approval of the manuscript; or decision to submit the manuscript for publication.

\section{Disclosure}

The authors report no disclosures relevant to the manuscript. Go to Neurology.org/N for full disclosures.

\section{Publication history}

Received by Neurology August 8, 2018. Accepted in final form November 13, 2018.

\section{Appendix Authors}

\begin{tabular}{|c|c|c|c|}
\hline Name & Location & Role & Contribution \\
\hline $\begin{array}{l}\text { Marios K. } \\
\text { Georgakis, } \\
\text { MD }\end{array}$ & $\begin{array}{l}\text { LMU } \\
\text { Munich, } \\
\text { Germany }\end{array}$ & Author & $\begin{array}{l}\text { Concept and design; data } \\
\text { acquisition, analysis, and } \\
\text { interpretation of data; statistical } \\
\text { analysis; drafting of the } \\
\text { manuscript; critical revision of the } \\
\text { manuscript for intellectual conten }\end{array}$ \\
\hline
\end{tabular}

\begin{tabular}{|c|c|c|c|}
\hline $\begin{array}{l}\text { Marco } \\
\text { Duering, } \\
\text { MD }\end{array}$ & $\begin{array}{l}\text { LMU } \\
\text { Munich, } \\
\text { Germany }\end{array}$ & Author & $\begin{array}{l}\text { Data acquisition, analysis, and } \\
\text { interpretation of data; critical } \\
\text { revision of the manuscript for } \\
\text { intellectual content }\end{array}$ \\
\hline $\begin{array}{l}\text { Joanna M. } \\
\text { Wardlaw, } \\
\text { MD }\end{array}$ & $\begin{array}{l}\text { University } \\
\text { of } \\
\text { Edinburgh, } \\
\text { UK }\end{array}$ & Author & $\begin{array}{l}\text { Data acquisition, analysis, and } \\
\text { interpretation of data; critical } \\
\text { revision of the manuscript for } \\
\text { intellectual content }\end{array}$ \\
\hline
\end{tabular}

\begin{tabular}{|c|c|c|c|}
\hline $\begin{array}{l}\text { Martin } \\
\text { Dichgans, } \\
\text { MD }\end{array}$ & $\begin{array}{l}\text { LMU } \\
\text { Munich, } \\
\text { Germany }\end{array}$ & Author & $\begin{array}{l}\text { Concept and design; data } \\
\text { acquisition, analysis, and } \\
\text { interpretation of data; drafting of } \\
\text { the manuscript; critical revision } \\
\text { of the manuscript for intellectual } \\
\text { content }\end{array}$ \\
\hline
\end{tabular}

\section{References}

1. Wardlaw JM, Smith EE, Biessels GJ, et al. Neuroimaging standards for research into small vessel disease and its contribution to ageing and neurodegeneration. Lancet Neurol 2013;12:822-838.

2. Wardlaw JM, Smith C, Dichgans M. Mechanisms of sporadic cerebral small vessel disease: insights from neuroimaging. Lancet Neurol 2013;12:483-497.

3. Pantoni L. Cerebral small vessel disease: from pathogenesis and clinical characteristics to therapeutic challenges. Lancet Neurol 2010;9:689-701.

4. de Leeuw FE, de Groot JC, Achten E, et al. Prevalence of cerebral white matter lesions in elderly people: a population based magnetic resonance imaging study: The Rotterdam Scan Study. J Neurol Neurosurg Psychiatry 2001;70:9-14.
5. Morris Z, Whiteley WN, Longstreth WT Jr, et al. Incidental findings on brain magnetic resonance imaging: systematic review and meta-analysis. BMJ 2009;339: b3016.

6. Maillard P, Seshadri S, Beiser A, et al. Effects of systolic blood pressure on whitematter integrity in young adults in the Framingham Heart Study: a cross-sectional study. Lancet Neurol 2012;11:1039-1047.

7. Staals J, Makin SD, Doubal FN, Dennis MS, Wardlaw JM. Stroke subtype, vascular risk factors, and total MRI brain small-vessel disease burden. Neurology 2014;83: 1228-1234.

8. Debette S, Markus HS. The clinical importance of white matter hyperintensities on brain magnetic resonance imaging: systematic review and meta-analysis. BMJ 2010; 341:c3666.

9. Rensma SP, van Sloten TT, Launer LJ, Stehouwer CDA. Cerebral small vessel disease and risk of incident stroke, dementia and depression, and all-cause mortality: a systematic review and meta-analysis. Neurosci Biobehav Rev 2018;90:164-173.

10. Inzitari D, Pracucci G, Poggesi A, et al. Changes in white matter as determinant of global functional decline in older independent outpatients: three year follow-up of LADIS (leukoaraiosis and disability) study cohort. BMJ 2009;339:b2477.

11. Wen W, Sachdev PS. Extent and distribution of white matter hyperintensities in stroke patients: the Sydney Stroke Study. Stroke 2004;35:2813-2819.

12. Leys D, Englund E, Del Ser T, et al. White matter changes in stroke patients: relationship with stroke subtype and outcome. Eur Neurol 1999;42:67-75.

13. Powers WJ, Rabinstein AA, Ackerson T, et al. 2018 guidelines for the early management of patients with acute ischemic stroke: a guideline for healthcare professionals from the American Heart Association/American Stroke Association. Stroke 2018;49:e46-e110.

14. Yang J, Wong A, Wang Z, et al. Risk factors for incident dementia after stroke and transient ischemic attack. Alzheimers Dement 2015;11:16-23.

15. Sachdev PS, Brodaty H, Valenzuela MJ, et al. Clinical determinants of dementia and mild cognitive impairment following ischaemic stroke: the Sydney Stroke Study. Dement Geriatr Cogn Disord 2006;21:275-283.

16. Kandiah N, Chander RJ, Lin X, et al. Cognitive impairment after mild stroke: development and validation of the SIGNAL2 risk score. J Alzheimers Dis 2016;49: 1169-1177.

17. IST-3 Collaborative Group. Association between brain imaging signs, early and late outcomes, and response to intravenous alteplase after acute ischaemic stroke in the third International Stroke Trial (IST-3): secondary analysis of a randomised controlled trial. Lancet Neurol 2015;14:485-496.

18. Ryu WS, Woo SH, Schellingerhout D, et al. Stroke outcomes are worse with larger leukoaraiosis volumes. Brain 2017;140:158-170.

19. Kongbunkiat K, Wilson D, Kasemsap N, et al. Leukoaraiosis, intracerebral hemorrhage, and functional outcome after acute stroke thrombolysis. Neurology 2017;88:638-645.

20. Lau KK, Li L, Schulz U, et al. Total small vessel disease score and risk of recurrent stroke: validation in 2 large cohorts. Neurology 2017;88:2260-2267.

21. Ntaios G, Lip GY, Lambrou D, et al. Leukoaraiosis and stroke recurrence risk in patients with and without atrial fibrillation. Neurology 2015;84:1213-1219.

22. van Swieten JC, Kappelle LJ, Algra A, van Latum JC, Koudstaal PJ, van Gijn J. Hypodensity of the cerebral white matter in patients with transient ischemic attack or minor stroke: influence on the rate of subsequent stroke: Dutch TIA Trial Study Group. Ann Neurol 1992;32:177-183.

23. Putaala J, Haapaniemi E, Kurkinen M, Salonen O, Kaste M, Tatlisumak T. Silent brain infarcts, leukoaraiosis, and long-term prognosis in young ischemic stroke patients. Neurology 2011;76:1742-1749.

24. Curtze S, Melkas S, Sibolt G, et al. Cerebral computed tomography-graded white matter lesions are associated with worse outcome after thrombolysis in patients with stroke. Stroke 2015;46:1554-1560.

25. Oksala NK, Oksala A, Pohjasvaara T, et al. Age related white matter changes predict stroke death in long term follow-up. J Neurol Neurosurg Psychiatry 2009;80:762-766.

26. Stroup DF, Berlin JA, Morton SC, et al. Meta-analysis of observational studies in epidemiology: a proposal for reporting: Meta-analysis of Observational Studies in Epidemiology (MOOSE) group. JAMA 2000;283:2008-2012.

27. Moher D, Liberati A, Tetzlaff J, Altman DG, Group P. Preferred reporting items for systematic reviews and meta-analyses: the PRISMA statement. PLoS Med 2009;6: e1000097.

28. Ferguson KJ, Cvoro V, MacLullich AMJ, et al. Visual rating scales of white matter hyperintensities and atrophy: comparison of computed tomography and magnetic resonance imaging. J Stroke Cerebrovasc Dis 2018;27:1815-1821.

29. Simoni M, Li L, Paul NL, et al. Age- and sex-specific rates of leukoaraiosis in TIA and stroke patients: population-based study. Neurology 2012;79:1215-1222.

30. New PW, Buchbinder R. Critical appraisal and review of the Rankin scale and its derivatives. Neuroepidemiology 2006;26:4-15.

31. Sulter G, Steen C, De Keyser J. Use of the Barthel index and modified Rankin Scale in acute stroke trials. Stroke 1999;30:1538-1541.

32. Synhaeve NE, Schaapsmeerders P, Arntz RM, et al. Cognitive performance and poor long-term functional outcome after young stroke. Neurology 2015;85:776-782.

33. Wells GA, Shea B, O'Connell D, Peterson J, Welch V, Losos M. The NewcastleOttawa Scale (NOS) for assessing the quality of nonrandomized studies in metaanalyses. [online]. Available at: ohri.ca/programs/clinical_epidemiology/oxford.asp. Accessed June7, 2018.

34. Hamling J, Lee P, Weitkunat R, Ambühl M. Facilitating meta-analyses by deriving relative effect and precision estimates for alternative comparisons from a set of estimates presented by exposure level or disease category. Stat Med 2008;27: 954-970. 
35. Belbasis L, Bellou V, Evangelou E, Ioannidis JP, Tzoulaki I. Environmental risk factors and multiple sclerosis: an umbrella review of systematic reviews and meta-analyses. Lancet Neurol 2015;14:263-273.

36. Egger M, Davey Smith G, Schneider M, Minder C. Bias in meta-analysis detected by a simple, graphical test. BMJ 1997;315:629-634.

37. Duval S, Tweedie R. Trim and fill: a simple funnel-plot-based method of testing and adjusting for publication bias in meta-analysis. Biometrics 2000;56:455-463.

38. Hartemink N, Boshuizen HC, Nagelkerke NJ, Jacobs MA, van Houwelingen HC. Combining risk estimates from observational studies with different exposure cutpoints: a meta-analysis on body mass index and diabetes type 2. Am J Epidemiol 2006; 163:1042-1052.

39. Orsini N, Li R, Wolk A, Khudyakov P, Spiegelman D. Meta-analysis for linear and nonlinear dose-response relations: examples, an evaluation of approximations, and software. Am J Epidemiol 2012;175:66-73.

40. Kapeller P, Barber R, Vermeulen RJ, et al. Visual rating of age-related white matter changes on magnetic resonance imaging: scale comparison, interrater agreement, and correlations with quantitative measurements. Stroke 2003;34:441-445.

41. Henninger N, Khan MA, Zhang J, Moonis M, Goddeau RP Jr. Leukoaraiosis predicts cortical infarct volume after distal middle cerebral artery occlusion. Stroke 2014;45: 689-695.

42. Helenius J, Mayasi Y, Henninger N. White matter hyperintensity lesion burden is associated with the infarct volume and 90-day outcome in small subcortical infarcts. Acta Neurol Scand 2017;135:585-592.
43. Ay H, Arsava EM, Rosand J, et al. Severity of leukoaraiosis and susceptibility to infarct growth in acute stroke. Stroke 2008;39:1409-1413.

44. Brown WR, Moody DM, Thore CR, Challa VR, Anstrom JA. Vascular dementia in leukoaraiosis may be a consequence of capillary loss not only in the lesions, but in normal-appearing white matter and cortex as well. J Neurol Sci 2007;257: $62-66$.

45. Shi Y, Thrippleton MJ, Makin SD, et al. Cerebral blood flow in small vessel disease: a systematic review and meta-analysis. J Cereb Blood Flow Metab 2016;36: $1653-1667$.

46. Tsivgoulis G, Zand R, Katsanos AH, et al. Risk of symptomatic intracerebral hemorrhage after intravenous thrombolysis in patients with acute ischemic stroke and high cerebral microbleed burden: a meta-analysis. JAMA Neurol 2016;73:675-683.

47. Charidimou A, Pasi M, Fiorelli M, et al. Leukoaraiosis, cerebral hemorrhage, and outcome after intravenous thrombolysis for acute ischemic stroke: a meta-analysis (v1). Stroke 2016;47:2364-2372.

48. Brickman AM, Siedlecki KL, Muraskin J, et al. White matter hyperintensities and cognition: testing the reserve hypothesis. Neurobiol Aging 2011;32:1588-1598.

49. Valdes Hernandez Mdel C, Booth T, Murray C, et al. Brain white matter damage in aging and cognitive ability in youth and older age. Neurobiol Aging 2013;34: $2740-2747$.

50. Duering M, Righart R, Wollenweber FA, Zietemann V, Gesierich B, Dichgans M. Acute infarcts cause focal thinning in remote cortex via degeneration of connecting fiber tracts. Neurology 2015;84:1685-1692. 